\title{
Otimização estrutural das paredes de silos metálicos prismáticos com a utilização do MS Excel Solver
}

\author{
Structural optimization of metallic prismatic \\ silos with MS Excel Solver
}

Moacir Kripka ${ }^{1}$; Paulo Agostinho Guerra ${ }^{2}$

Resumo

O presente trabalho tem por objetivo apresentar a aplicação do Microsoft Excel Solver na otimização de paredes de silos metálicos prismáticos enrijecidas, conformadas com ondas horizontais em ziguezague e trapezoidal, buscando a construção de uma ferramenta auxiliar ao projetista estrutural. Alguns resultados são apresentados com o intuito de ilustrar o emprego do Solver, evidenciando a economia resultante da aplicação da formulação desenvolvida.

Palavras-chave: Silos. Otimização. Estruturas.

\begin{abstract}
The aim of this work is to present the application of Microsoft Excel Solver to the optimization of stiffened walls of metallic prismatic silos, profiled in trapezoidal and zigzag sections, seeking the construction of an auxiliary tool to structural designers. Some numerical results are presented in order illustrate the use of Solver, as well as to show the significant economy achieved with to the developed formulation.
\end{abstract}

Key words: Silos. Optimization. Structures.

\footnotetext{
Professor do Programa de Pós-Graduação em Engenharia da Universidade de Passo Fundo - PPGEng/UPF. E-mail: mkripka@, upf.br

2 Mestre em Engenharia pelo Programa de Pós-Graduação em Engenharia da Universidade de Passo Fundo - PPGEng/UPF.
} 


\section{Introdução}

Nos últimos anos, dada a evolução dos processos de industrialização do aço (tanto pelo avanço tecnológico dos equipamentos como dos materiais) e do conhecimento, foi possível a padronização e a modulação da fabricação das estruturas de aço para os mais diversos fins. É o caso dos silos circulares, totalmente fabricados em painéis modulados que são montados com rapidez por uniões parafusadas no local definitivo da planta de produção. Também é notória a modernização alcançada pelos equipamentos computacionais que estimulam o desenvolvimento de novas metodologias de análise estrutural, mais consistentes e eficientes para representar estruturas reais. Em contraposição, os estudos sobre silos prismáticos são escassos e limitados. De fato, poucos pesquisadores dedicaram parte de seus trabalhos a esse estudo, destacandose: Ravenet (1977), Troitsky (1988) e, no Brasil, os trabalhos de Calil e Nascimento (1997).

Silos retangulares não são intrinsecamente a forma estrutural mais eficiente (TRAHAIR, 1985). No entanto, reduzidos custos de fabricação e completo aproveitamento do espaço disponível os tornam economicamente atraentes. Para uma mesma altura, silos quadrados propiciam $27 \%$ a mais de armazenagem em comparação com silos circulares de diâmetro igual ao comprimento do lado do silo quadrado. Em geral, os silos retangulares são menos sensíveis a cargas assimétricas e os efeitos estruturais são mais fáceis de serem calculados (GAYLORD JUNIOR; GAYLORD, 1984).
O sistema de construção de silos retangulares consiste na pré-fabricação de painéis metálicos para as paredes que, mediante a conformação horizontal, ofereçam a resistência suficiente para resistir às pressões exercidas pelos produtos armazenados.

Não obstante as diversas soluções de conformação das paredes propostas até o momento, somente dois tipos progrediram e são utilizados nas construções de grandes silos (RAVENET, 1977): o de conformação trapezoidal (para armazenamento de produtos granulares de fácil descarga) e o de conformação ziguezague (armazenamento de produtos pulverulentos coesivos). Para manter a rigidez da chapa dobrada, colocam-se platibandas soldadas em suas extremidades (Figura 1).

As ondas horizontais conformadas em forma trapezoidal ou ziguezague têm como objetivo aumentar a inércia das paredes e, em conseqüência, diminuir a quantidade de material empregado na fabricação dos painéis. Por outro lado, o atrito e as pressões atuantes sobre as paredes dependem das características físicas e mecânicas do produto (peso específico, coesão e ângulo de atrito interno), da geometria da célula de armazenamento (seção transversal e altura) e do tipo de fluxo (centrado ou excêntrico). Por conseguinte, a intensidade das ações será função dessas variáveis, e a resistência às solicitações definida pelo ângulo de inclinação da onda horizontal com o plano vertical, o qual deverá ser maior que o ângulo de atrito interno do produto armazenado. Busca-se, desta forma, evitar o acúmulo de produto nas paredes e, em conseqüência, que as mesmas sejam contaminadas por fungos e bactérias. 

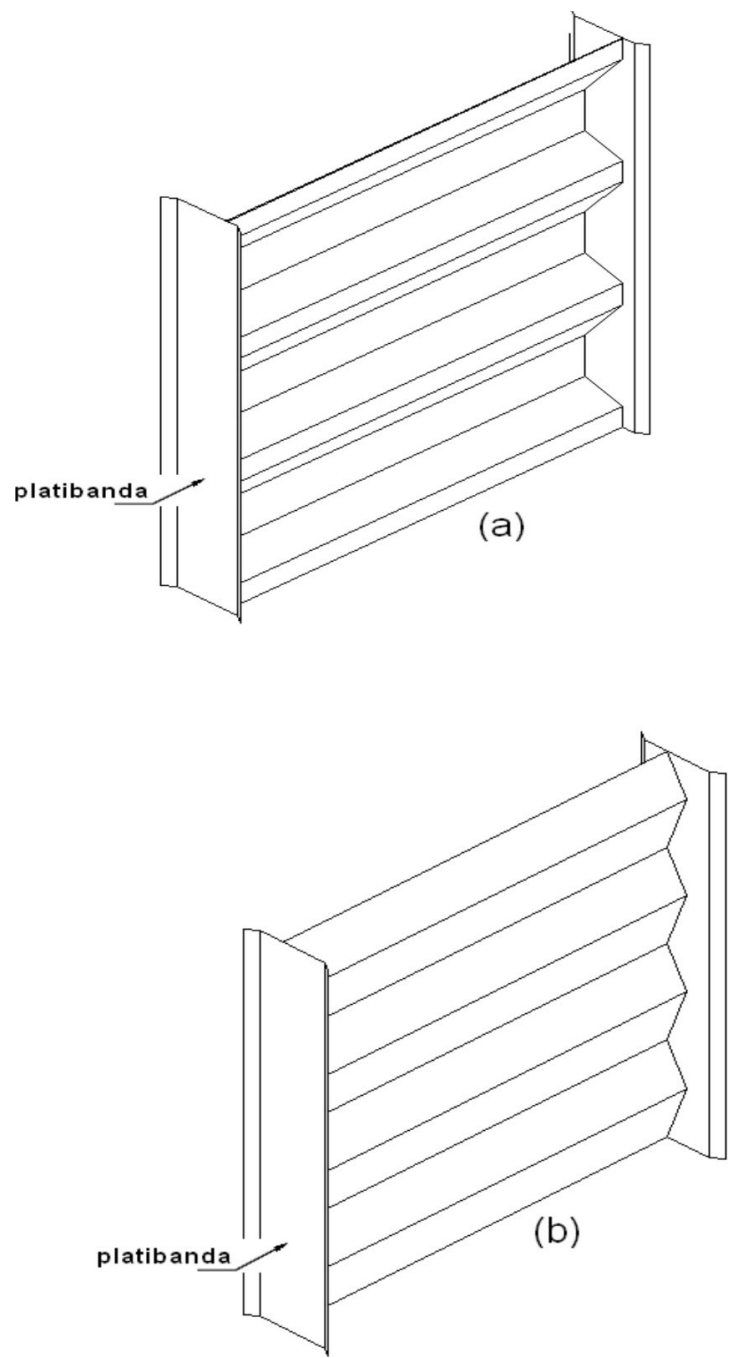

Figura 1. Conformação trapezoidal (a) e em ziguezague (b)

Dentro desse contexto, sendo as paredes dos silos prismáticos o elemento determinante do maior consumo de aço, objetivou-se o desenvolvimento de um sistema de apoio ao projeto de silos metálicos prismáticos, para a avaliação do desempenho estrutural das paredes enrijecidas, conformadas com ondas horizontais em forma trapezoidal e ziguezague. Pela aplicação da formulação desenvolvida a um software de otimização, o peso mínimo é selecionado para a função objetivo, uma vez que o custo do aço empregado na fabricação das células é o fator preponderante no valor final do silo. Assim, minimizando-se o peso do aço, por conseqüência, reduz-se o custo final das células e das fundações.

O crescente emprego das técnicas de otimização à resolução de problemas práticos tem sido impulsionado pela grande economia proporcionada, a qual é evidenciada pela farta produção científica gerada. Normalmente, no entanto, uma vez formulado o problema, o mesmo é resolvido com a utilização de softwares comerciais ou, alternativamente, pela implementação computacional segundo alguma técnica específica. Enquanto os softwares comerciais, de forma geral, apresentam elevado custo de aquisição, o desenvolvimento de um programa, por sua vez, exige um conhecimento razoável tanto das técnicas como de uma linguagem de programação.

O presente trabalho tem por objetivo apresentar a aplicação do Microsoft Excel Solver ao problema descrito. O Microsoft Excel Solver consiste numa ferramenta acessível e de fácil utilização, fundamentada em técnica de otimização não-linear conhecida como Gradiente Reduzido Generalizado.

Nas seções seguintes deste trabalho são apresentadas a formulação desenvolvida e sua implementação. Alguns resultados são apresentados com o intuito de evidenciar a economia resultante da aplicação da ferramenta desenvolvida.

\section{Formulação do Problema de Otimização}

Visando a avaliação do desempenho estrutural das paredes de silos prismáticos enrijecidas, optouse por formular o dimensionamento das paredes como um problema de otimização estrutural. Esta abordagem foi adotada com o objetivo de propiciar a efetiva comparação entre as diversas configurações, quando submetidas a uma mesma condição de carregamento e vinculação, bem como a construção de uma ferramenta de fácil utilização. Assim, para cada configuração, busca-se a determinação das dimensões que conduzam a maior economia. 
O objetivo em um problema de otimização é localizar os extremos de uma determinada função, isto é, determinar o conjunto de variáveis das quais tal função é dependente, de modo a encontrar seu valor máximo ou mínimo.

Matematicamente, um problema de otimização não-linear e sujeito a restrições pode ser enunciado da seguinte forma (VANDERPLAATS, 1984):

Minimizar (ou maximizar):

$$
F(X)
$$

Sujeito a:

$$
\begin{aligned}
& g_{j}(X) \leq 0 \quad \mathrm{j}=1, \mathrm{~m} \\
& h_{k}(X)=0 \quad \mathrm{k}=1,1 \\
& X_{i}^{l} \leq X \leq X_{i}^{u} \quad \mathrm{i}=1, \mathrm{n}
\end{aligned}
$$

Define-se $X$ como o vetor que contém as variáveis de projeto. A função objetivo $\mathrm{F}(\mathrm{X})$ é fornecida pela Equação (1), e as funções de restrição, definidas pelas Equações (2) e (3), podendo ser lineares ou não. As equações envolvidas podem ser explícitas ou implícitas com relação a $X$. A Equação (4) define os limites para as variáveis de projeto e, subseqüentemente, refere-se às restrições laterais. Ainda que restrições laterais possam ser incluídas em restrições de desigualdade dada pela Equação (2), é usual tratá-las separadamente, uma vez que definem a região de busca do ótimo.

No presente estudo, para ambas as configurações o problema foi formulado tendo como variáveis de projeto a espessura $(t)$, o ângulo da inclinação do segmento da onda com a horizontal $(\theta)$ e o comprimento do segmento da onda $\left(L_{c}\right)$, objetivando a minimização do peso por metro de seção vertical de parede. Estas variáveis estão identificadas na Figura 2, para uma seção trapezoidal.

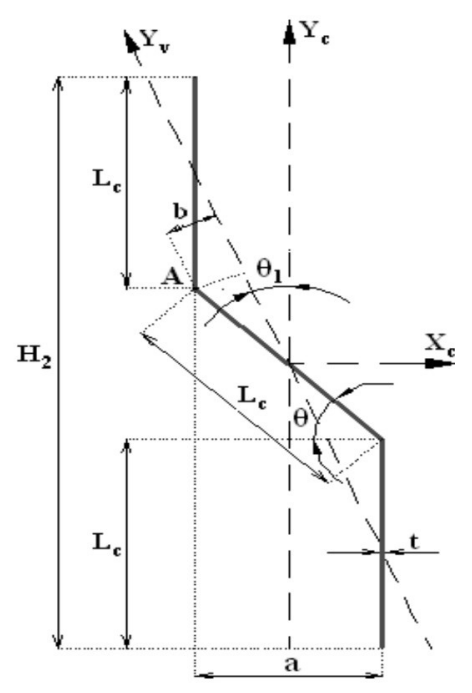

Figura 2. Seção da onda trapezoidal

Para a configuração em ziguezague a função objetivo foi escrita como:

$$
f_{(o)}=\frac{t}{\operatorname{sen} \theta} \cdot \gamma
$$

e para a trapezoidal:

$$
f_{(o)}=\frac{2 \cdot t}{1+\operatorname{sen} \theta} \cdot \gamma
$$

Em ambas as expressões anteriores, $\gamma$ representa o peso específico do aço. Observa-se que, pelo fato do número de segmentos da onda por metro de altura do silo ser função também do ângulo da inclinação de cada segmento, a variável $L_{c}$ não aparece de forma explícita na função objetivo.

As possíveis soluções do problema devem atender a um conjunto de condições (ou restrições), segundo as quais as tensões atuantes $\sigma_{y}, \sigma_{v}$ e $\sigma_{l}$, correspondentes às flexões geral, oblíqua e local, respectivamente, não devem ultrapassar a tensão admissível $\sigma_{a d m}$ : 


$$
\begin{gathered}
\sigma_{Y c} \leq \sigma_{a d m} \\
\sigma_{Y v} \leq \sigma_{a d m} \\
\sigma_{l} \leq \sigma_{a d m}
\end{gathered}
$$

Às variáveis foram impostas também restrições laterais, as quais definem limites inferiores e/ou superiores para os valores que podem ser assumidos em cada uma delas.

Por questão de concisão, o presente trabalho omite a formulação correspondente à forma de determinação das tensões indicadas nas expressões anteriores. Uma descrição detalhada dessa formulação, bem como da forma de obtenção das funções objetivo e restrições, pode ser obtida em Guerra (2006) e em Kripka e Guerra (2008).

\section{Implementação da Formulação no Solver}

O problema formulado no item anterior é classificado como um problema não-linear, restrito e com variáveis contínuas, o qual foi resolvido de forma conveniente através do método do gradiente reduzido generalizado, empregado no Solver. Esse método, referenciado como GRG2, foi desenvolvido por Leon Lasdon, da Universidade do
Texas em Austin e Allan Waren, da Universidade Estadual de Cleveland. Maiores informações sobre o processo interno de solução do Solver estão disponíveis em: http://office.microsoft.com/pt-br/ excel/ HA0112459 51046 .aspx.

O recurso Solver pode ser acessado no Microsoft Excel, na caixa de seleção Solver em Suplementos do menu Ferramentas.

Nesta seção, são apresentadas a descrição do software e as instruções necessárias para sua utilização na resolução de problemas de otimização. Para usar o Solver é necessário que se crie um modelo que especifique: as variáveis de decisão, a função objetivo e as restrições.

\section{Variáveis de decisão (células variáveis):}

Os parâmetros a serem introduzidos no problema de otimização podem ser quantidades, as quais estão sujeitas a variações ou sob o controle do usuário. Esses parâmetros são aplicados para as variáveis de decisão e aparecem na planilha eletrônica nas células variáveis, que são aquelas que o Solver irá alterar automaticamente para maximizar ou minimizar o objetivo ou célula de destino. Essas células são registradas na caixa de edição de células variáveis do quadro de diálogo dos Parâmetros do Solver (Figura 3).

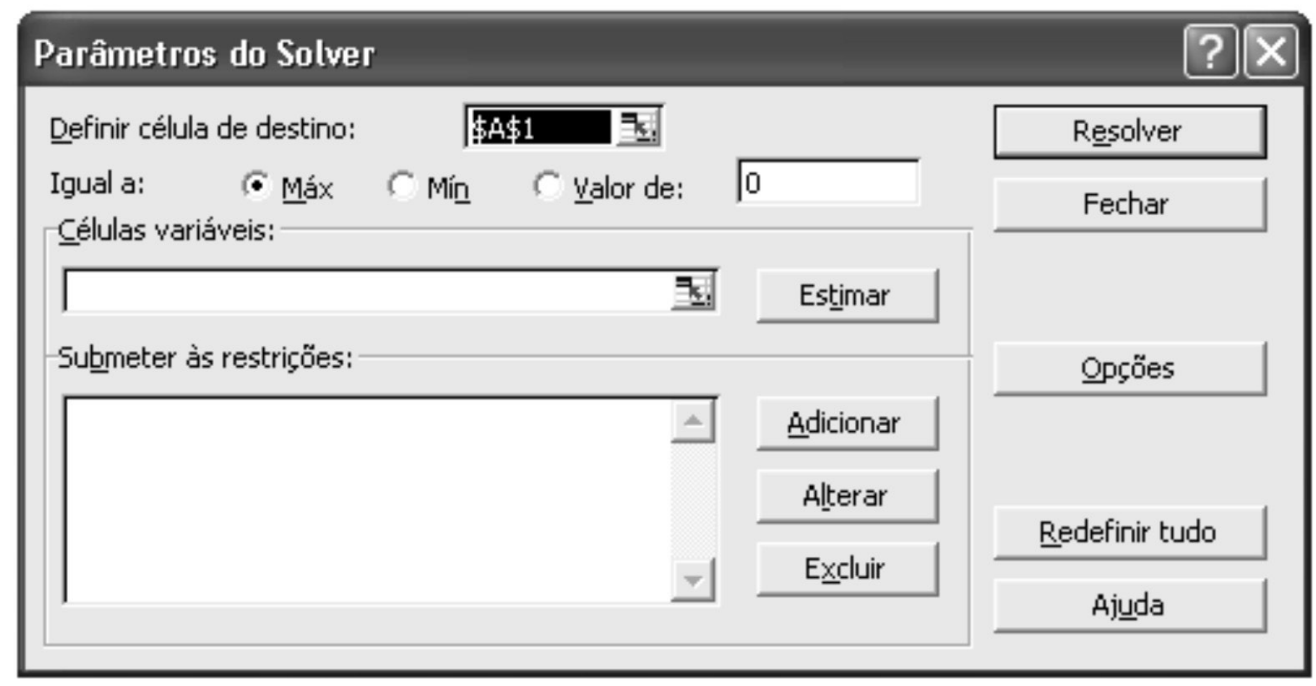

Figura 3. Quadro de diálogo dos Parâmetros do Solver. 
Função objetivo (célula de destino):

A função objetivo é inserida na caixa de edição definir célula de destino do quadro diálogo dos Parâmetros do Solver. No caso de no modelo do Solver não se ter nada para maximizar ou minimizar, a célula de destino ficará vazia. Nesta situação, o Solver irá simplesmente encontrar uma solução que satisfaça às restrições.

Planilhas eletrônicas padrão de otimização também permitem a entrada de um valor específico, o qual deve ser atingido pela função objetivo ou célula de destino. Essa característica permite a compatibilidade com a planilha Atingir Metas (Goal Seek) ou Regressão (Back Solver) e está disponível no menu de comandos do Excel, que permite procurar um valor especifico para a célula pelo ajustamento do valor de uma outra célula da qual a primeira é dependente.

\section{Restrições:}

São limites impostos às células variáveis. $\mathrm{O}$ Solver considera que uma restrição é satisfeita se a condição que esta restrição especifica é verdadeira, observados pequenos limites de tolerância. Os valores de tolerância, precisão do Solver e métodos numéricos empregados na solução de um problema de otimização estão contidos no quadro de Opções do Solver.

Uma solução (valores para as variáveis de decisão) para as quais todas as restrições no modelo são satisfeitas é denominada de solução factivel. $\mathrm{Na}$ seqüência, o Solver, primeiro, encontra uma solução factível e, após, procura melhorá-la, substituindo as variáveis de decisão de uma solução factível para outra solução factível, até que a função objetivo alcance um máximo ou mínimo. Esse processo é denominado de solução ótima. A solução ótima pode ser global, quando não existe outra solução factível com valores mais adequados para a função objetivo, ou local, quando não existe outra solução com melhores valores na vizinhança. O Solver é programado para encontrar soluções ótimas, em condições ideais o ótimo global, porém nem sempre isso é possível. Em muitos casos, uma boa solução pode ser suficiente, ou seja, uma solução melhor que a anterior.

\section{Resultados Obtidos}

A partir da implementação das formulações desenvolvidas no Microsoft Excel Solver, foram efetuadas diversas simulações para paredes conformadas com a seção da onda trapezoidal e ziguezague. Essas simulações objetivaram, num primeiro momento, demonstrar a potencial economia obtida com a aplicação de técnicas de otimização ao problema proposto. Adicionalmente, puderam ser efetivamente comparadas as conformações estudadas, obtendo-se a configuração ótima (e o peso correspondente) para cada uma quando submetidas às mesmas condições de carregamento e resistência.

No presente trabalho são apresentados alguns dos resultados obtidos, selecionados com o objetivo de ilustrar a aplicação do Solver. A estrutura apresentada neste exemplo (Figura 4) consiste num painel de conformação trapezoidal proposto por Ravenet (1992) para uma célula com as seguintes características: largura de 4,25 m, altura de $25 \mathrm{~m}$, distância entre colunas $(L)$ igual a $2 \mathrm{~m}$, largura da platibanda de ancoragem do perfil conformado $a$ $=21 \mathrm{~cm}$ e ângulo mínimo que forma o segmento inclinado da chapa conformada com o eixo baricêntrico $X_{c} \theta=45^{\circ}$. O produto armazenado é um cereal com densidade de $8,40 \mathrm{kN} / \mathrm{m}^{3}$ e tensão admissível do aço $\sigma_{a d m}=161,81 \mathrm{MPa}$. As pressões $P_{h}$ utilizadas para o dimensionamento são as da curva de pressões laterais de Platonov (RAVENET, 1977). 

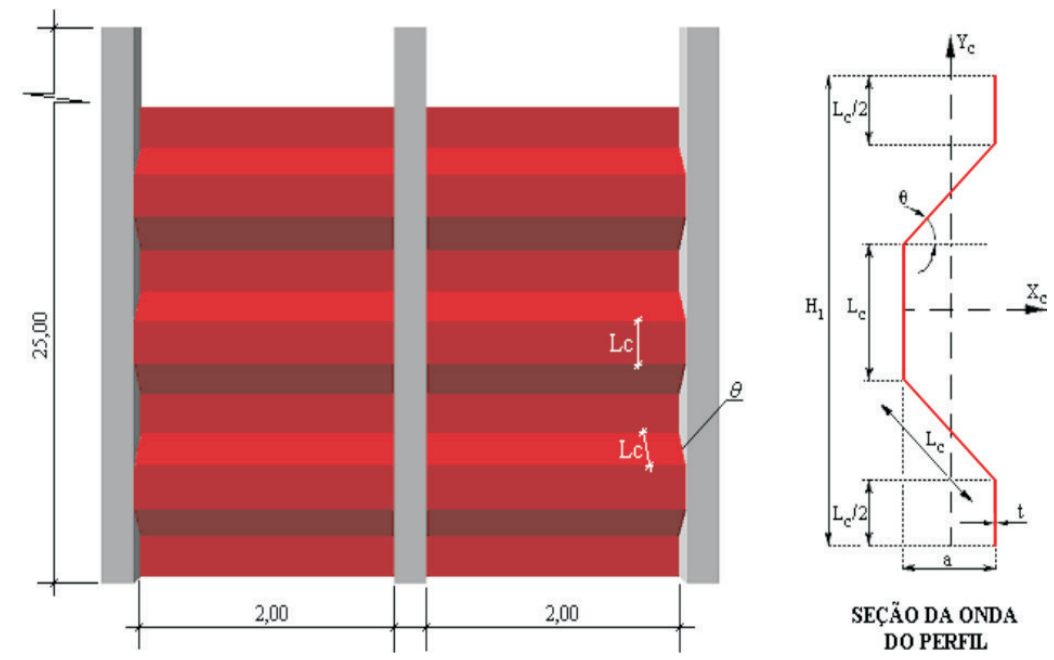

Figura 4. Vista frontal da parede do silo e seção transversal da onda do perfil.

A Figura $\mathbf{5}$ apresenta a construção do modelo na planilha eletrônica para a conformação trapezoidal, bem como os resultados para a pressão horizontal na cota $25 \mathrm{~m}$ (medida do topo até a base da parede). Para se obter o dimensionamento otimizado para as demais cotas basta fornecer a pressão horizontal em cada uma delas e executar o Solver.
A coluna Modelo indica o atendimento às restrições para os valores atuais das variáveis segundo a tolerância predefinida. No caso ilustrado na Figura 5, o falso corresponde à violação da tensão $\sigma_{\mathrm{v}}$ na sexta casa decimal. Assim, cabe ao usuário aceitar (ou não) o resultado com tal grau de violação.

\begin{tabular}{|c|c|c|c|c|}
\hline \multicolumn{5}{|l|}{ Objetivo: } \\
\hline \multicolumn{5}{|c|}{$\begin{array}{l}\text { Encontrar os valores de } t \text {, } L c \text { e } \theta \text { que minimizam o peso } P \text { do painel em conformação } \\
\text { trapezoidal. }\end{array}$} \\
\hline \multicolumn{2}{|r|}{ Função Objetivo } & \multicolumn{2}{|c|}{ Dados do Projeto } & Modelo \\
\hline \multirow[t]{3}{*}{$P=$} & $0,394952182 \mathrm{kN} / \mathrm{m}$ & $\mathrm{P}_{\mathrm{h}}=$ & $0,04223724 \mathrm{MPa}$ & 0,394952182 \\
\hline & & $\mathrm{L}=$ & $2 \mathrm{~m}$ & 3 \\
\hline & & $\sigma_{\mathrm{adm}}=$ & $161,809700 \mathrm{MPa}$ & VERDADEIRO \\
\hline \multicolumn{2}{|r|}{ Variáveis diretas } & \multicolumn{2}{|c|}{ Restrições de comportamento } & VERDADEIRO \\
\hline $\mathrm{t}=$ & $0,00438 \mathrm{~m}$ & $\sigma_{\mathrm{Yv}}=$ & $161,8097000 \mathrm{MPa}$ & VERDADEIRO \\
\hline$\theta=$ & 45,000000 graus & $\sigma_{\mathrm{Yc}}=$ & $47,61 \mathrm{MPa}$ & VERDADEIRO \\
\hline $\mathrm{L}_{\mathrm{c}}=$ & $0,2969848 \mathrm{~m}$ & $\sigma_{1}=$ & $97,13 \mathrm{MPa}$ & VERDADEIRO \\
\hline \multicolumn{2}{|c|}{ Propriedades do perfil } & \multicolumn{2}{|c|}{ Restrições laterais } & VERDADEIRO \\
\hline $\mathrm{W}_{\mathrm{y}}=$ & $3,63 \mathrm{E}-04 \mathrm{~m}^{3}$ & $\mathrm{t} \geq$ & $0,001 \mathrm{~m}$ & FALSO \\
\hline $\mathrm{I}_{\mathrm{xc}}=$ & $1,88 \mathrm{E}-04 \mathrm{~m}^{4}$ & $t \leq$ & $0,008 \mathrm{~m}$ & VERDADEIRO \\
\hline $\mathrm{I}_{\mathrm{yc}}=$ & $3,33 \mathrm{E}-05 \mathrm{~m}^{4}$ & $\theta \geq$ & 45 graus & VERDADEIRO \\
\hline$I_{x y}=$ & $-7,33 \mathrm{E}-05 \mathrm{~m}^{4}$ & $\theta \leq$ & 90 graus & 100 \\
\hline$\theta_{1}=$ & 21,69240191 graus & $\mathrm{L}_{\mathrm{c}} \geq$ & $0,155563492 \mathrm{~m}$ & \\
\hline$I_{v}=$ & $4,11 \mathrm{E}-06 \mathrm{~m}^{4}$ & $\mathrm{~L}_{\mathrm{c}} \leq$ & $0,296984848 \mathrm{~m}$ & \\
\hline $\mathrm{W}_{\mathrm{v}}=$ & $7,00 \mathrm{E}-05 \mathrm{~m}^{3}$ & & & \\
\hline
\end{tabular}

Figura 5. Planilha de dados - Conformação trapezoidal proposta por Ravenet para a cota $25 \mathrm{~m}$. 
As figuras seguintes apresentam os relatórios gerados pelo Solver para o Exemplo.

O relatório de resposta, ilustrado na Figura 6, apresenta os valores iniciais (originais) e finais das variáveis de projeto e os valores correspondentes da função objetivo, bem como informações sobre as restrições.A coluna transigência indica os valores das variáveis de folga e de excesso. Por exemplo, na segunda linha de $L_{c}$, o valor de transigência aponta de quanto o limite inferior atribuído a esse variável foi superado. Na linha imediatamente anterior o número zero aponta que o limite superior foi atingido. Em outras palavras, que a restrição é ativa no ponto, ou seja, a alteração no limite imposto para o valor máximo que a variável pode assumir acarreta na alteração da solução ótima para o problema. Todas as restrições ativas são indicadas pelo status agrupar.

\begin{tabular}{|c|c|c|c|c|c|}
\hline \multicolumn{6}{|c|}{$\begin{array}{l}\text { Microsoft Excel 9.0 Relatório de } \\
\text { Planilha: [modelo1A.xls]TRAP }\end{array}$} \\
\hline \multicolumn{6}{|c|}{ Célula de destino (Mín) } \\
\hline Célula & Nome & Valor original & Valor final & & \\
\hline$\$ B \$ 5$ & $\mathrm{P}$ & 0,541141544 & 0,394952169 & & \\
\hline \multicolumn{6}{|c|}{ Células ajustáveis } \\
\hline Célula & Nome & Valor original & Valor final & & \\
\hline$\$ B \$ 10$ & $\mathrm{t}$ & 0,00600 & 0,00438 & & \\
\hline$\$ B \$ 11$ & $\theta$ & 45,000000 & 45,000000 & & \\
\hline$\$ B \$ 12$ & $\mathrm{Lc}$ & 0,3000000 & 0,2969848 & & \\
\hline \multicolumn{6}{|l|}{ Restrições } \\
\hline Célula & Nome & Valor da célula & Fórmula & Status & Transigência \\
\hline$\$ B \$ 12$ & Lc & 0,2969848 & $\$ B \$ 12<=\$ F \$ 21$ & Agrupar & 0 \\
\hline$\$ B \$ 12$ & $\mathrm{Lc}$ & 0,2969848 & $\$ B \$ 12>=\$ F \$ 20$ & Sem agrupar & 0,1414214 \\
\hline$\$ F \$ 10$ & $\sigma \mathrm{V}$ & 161,81 & $\$ F \$ 10<=\$ F \$ 7$ & Sem agrupar & $5,34604 \mathrm{E}-06$ \\
\hline$\$ F \$ 11$ & $\sigma y$ & 47,61 & $\$ F \$ 11<=\$ F \$ 7$ & Sem agrupar & 114,1976376 \\
\hline$\$ F \$ 12$ & $\sigma \mathrm{l}$ & 97,13 & $\$ F \$ 12<=\$ F \$ 7$ & Sem agrupar & 64,67724437 \\
\hline$\$ B \$ 10$ & $\mathrm{t}$ & 0,00438 & $\$ B \$ 10<=\$ F \$ 17$ & Sem agrupar & 0,0036209 \\
\hline$\$ B \$ 10$ & $\mathrm{t}$ & 0,00438 & $\$ B \$ 10>=\$ F \$ 16$ & Sem agrupar & 0,00338 \\
\hline$\$ B \$ 11$ & $\theta$ & 45,000000 & $\$ B \$ 11<=\$ F \$ 19$ & Sem agrupar & 44,99999995 \\
\hline$\$ B \$ 11$ & $\theta$ & 45,000000 & $\$ B \$ 11>=\$ F \$ 18$ & Agrupar & 0,000000 \\
\hline
\end{tabular}

Figura 6. Relatório de resposta na cota 25 metros.

Na Figura 7 o Relatório de limites lista a célula de destino e as células ajustáveis com seus respectivos valores, limites inferior e superior e valores de destino. O limite inferior é o menor valor que a célula ajustável pode usar, mantendo todas as outras células ajustáveis fixas, e, ainda assim, atender às restrições. Analogamente, o limite superior é o valor mais alto. Observa-se que, pelo fato da espessura ser a única variável que não está ativa no ponto de ótimo, é também a única que pode ter seu valor alterado (no caso, aumentado) sem violar nenhuma restrição. No entanto, o peso da estrutura também seria aumentado.

A Figura 8 apresenta o cenário para uma solução inicial distinta (no caso infactível). A coluna valores atuais representa os valores das células no momento em que o relatório de resumo do cenário foi criado e as células variáveis para cada cenário estão destacadas em negrito. 
Microsoft Excel 9.0 Relatório de limites

Planilha: [modelo1A.xls]TRAP

\begin{tabular}{lcc}
\hline Célula & $\begin{array}{c}\text { Destino } \\
\text { Nome }\end{array}$ & Valor \\
\hline$\$ \mathrm{~B} \$ 5$ & $\mathrm{P}$ & 0,394952169 \\
\hline \multicolumn{3}{c}{ Ajustável } \\
Célula & Nome & Valor \\
\hline$\$ \mathrm{~B} \$ 10$ & $\mathrm{t}$ & 0,00438 \\
\hline $\mathrm{B} \$ 11$ & $\theta$ & 45,000000 \\
\hline B $\$ 12$ & $\mathrm{Lc}$ & 0,2969848 \\
\hline
\end{tabular}

\begin{tabular}{cr}
\hline $\begin{array}{c}\text { Inferior } \\
\text { Limite }\end{array}$ & \multicolumn{1}{c}{$\begin{array}{c}\text { Destino } \\
\text { Resultado }\end{array}$} \\
\hline 0,00438 & 0,39495 \\
\hline 45,000000 & 0,394952 \\
\hline 0,2969848 & 0,3949522 \\
\hline
\end{tabular}

\begin{tabular}{rr}
\hline $\begin{array}{c}\text { Superior } \\
\text { Limite }\end{array}$ & $\begin{array}{c}\text { Destino } \\
\text { Resultado }\end{array}$ \\
\hline 0,00800 & 0,72152 \\
\hline 45,000000 & 0,394952 \\
\hline 0,2969848 & 0,3949522 \\
\hline
\end{tabular}

Figura 7. Relatório de limites na cota 25 metros.

\begin{tabular}{rrrr}
\hline Resumo do cenário & & & \\
& Valores atuais: & RAVENET & TRAP \\
\hline Células variáveis: & & & \\
\hline \$B $\$ 10$ & 0,00438 & $\mathbf{0 , 0 0 6 0 0}$ & $\mathbf{1 , 0 0 0 0 0}$ \\
\$B $\$ 11$ & 45,000000 & $\mathbf{4 5 , 0 0 0 0 0 0}$ & $\mathbf{1 , 0 0 0 0 0 0}$ \\
\$B \$12 & 0,2969848 & $\mathbf{0 , 3 0 0 0 0 0 0}$ & $\mathbf{1 , 0 0 0 0 0 0 0}$ \\
\hline Células de resultado: & & & \\
\hline \$B $\$ 5$ & 0,394952169 & 0,541141544 & 151,3234418 \\
\$F\$10 & 161,81 & 117,08 & 0,15 \\
\$F\$11 & 47,61 & 34,50 & 0,10 \\
\$F\$12 & 97,13 & 52,80 & 0,02 \\
\$F\$13 & 21,69240189 & 21,72416857 & $-1,060037565$ \\
\hline
\end{tabular}

Figura 8. Resumo do cenário para a cota 25 metros.

$\mathrm{Na}$ Figura 6 os valores iniciais das células variáveis (coluna: Valor original) correspondem aos resultados obtidos por Ravenet dimensionando as paredes da célula pela teoria clássica. No entanto, para a obtenção da solução ótima partiu-se de várias soluções iniciais com o objetivo de verificar a convergência para um único ponto (exemplificando: a descrita pelo cenário TRAP da Figura 8). Dessa forma, para ambas as situações se observa a mesma solução listada na coluna valores atuais, indicando que o ótimo pode ser obtido independentemente dos valores inicialmente atribuídos às variáveis de projeto.

A Tabela 1 reproduz os resultados do painel dimensionado por Ravenet para diversas alturas ao longo da parede do silo. Na Tabela 2 são apresentados os resultados do modelo trapezoidal de Ravenet otimizado. O peso ótimo $P$, indicado na sexta coluna, para as cotas z entre 1,9 e 8,12 m é limitado pelas tensões $\sigma_{Y v}$ e $\sigma_{p}$; para a cota de 25 m, o ótimo e limitado unicamente pela tensão $\sigma_{Y v}$, conforme demonstra o resumo do cenário (Figura 8). 
Tabela 1 - Dimensionamento de Ravenet para conformação trapezoidal

\begin{tabular}{|c|c|c|c|c|c|c|c|c|}
\hline \multicolumn{9}{|c|}{ RESULTADOS DO DIMENSIONAMENTO DE RAVENET - (Ph para curva Platonov) } \\
\hline \multirow{2}{*}{$\begin{array}{c}\operatorname{cota} \mathrm{z} \\
\text { (m) }\end{array}$} & \multirow{2}{*}{$\begin{array}{c}\text { pressão } \\
\text { cálculo } \\
\mathrm{Ph} \\
(\mathrm{MPa})\end{array}$} & \multicolumn{7}{|c|}{ PAREDE EM CONFORMAÇÃO TRAPEZOIDAL L=2m e h=25m } \\
\hline & & $\begin{array}{l}\mathrm{Lc} \\
(\mathrm{m})\end{array}$ & $\begin{array}{c}\theta \\
\text { (graus) }\end{array}$ & $\underset{(\mathrm{m})}{\mathrm{t}}$ & $\begin{array}{c}\mathrm{P} \\
(\mathrm{kN} / \mathrm{m})\end{array}$ & $\begin{array}{c}\sigma \mathrm{Yv} \\
(\mathrm{MPa})\end{array}$ & $\begin{array}{c}\sigma \mathrm{Yc} \\
(\mathrm{MPa})\end{array}$ & $\begin{array}{c}\sigma \mathrm{l} \\
(\mathrm{MPa})\end{array}$ \\
\hline 1,90 & 0,019 & 0,3000 & 45 & 0,00300 & 0,271 & 104,24 & 30,74 & 94,29 \\
\hline 3,90 & 0,021 & 0,3000 & 45 & 0,00400 & 0,361 & 87,63 & 25,83 & 59,38 \\
\hline 8,12 & 0,038 & 0,3000 & 45 & 0,00500 & 0,451 & 125,35 & 36,94 & 67,89 \\
\hline 25,00 & 0,042 & 0,3000 & 45 & 0,00600 & 0,541 & 117,08 & 34,50 & 52,80 \\
\hline
\end{tabular}

Tabela 2 - Dimensionamento para conformação trapezoidal otimizada

\begin{tabular}{|c|c|c|c|c|c|c|c|c|}
\hline \multicolumn{10}{|c|}{ MODELO DE RAVENET OTIMIZADO } \\
\hline \multirow{2}{*}{$\begin{array}{c}\text { cota z } \\
(\mathrm{m})\end{array}$} & $\begin{array}{c}\text { pressão } \\
\text { cálculo } \\
\mathrm{Ph} \\
(\mathrm{MPa})\end{array}$ & $\begin{array}{c}\mathrm{Lc} \\
(\mathrm{m})\end{array}$ & $\begin{array}{c}\theta \\
\text { (graus) }\end{array}$ & $\begin{array}{c}\mathrm{t} \\
(\mathrm{m})\end{array}$ & $\begin{array}{c}\mathrm{P} \\
(\mathrm{kN} / \mathrm{m})\end{array}$ & $\begin{array}{c}\sigma \mathrm{Yv} \\
(\mathrm{MPa})\end{array}$ & $\begin{array}{c}\sigma \mathrm{Yc} \\
(\mathrm{MPa})\end{array}$ & $\begin{array}{c}\sigma \mathrm{l} \\
(\mathrm{MPa})\end{array}$ \\
\hline 1,90 & 0,019 & 0,2755 & 45 & 0,00210 & 0,190 & 161,81 & 47,04 & 161,81 \\
\hline 3,90 & 0,021 & 0,2834 & 45 & 0,00229 & 0,206 & 161,81 & 47,26 & 161,81 \\
\hline 8,12 & 0,038 & 0,2970 & 45 & 0,00391 & 0,353 & 161,81 & 47,62 & 161,81 \\
\hline 25,00 & 0,042 & 0,2970 & 45 & 0,00438 & 0,395 & 161,81 & 47,61 & 97,13 \\
\hline
\end{tabular}

A Tabela 3 apresenta o resumo dos resultados do dimensionamento realizado por Ravenet e os da otimização, bem como a economia de material em percentagem na coluna Redução do peso otimizado.
Tanto a Tabela 3 como a Figura 9 evidenciam a grande redução do material das paredes do silo, sendo que nos pontos estudados a economia variou entre $21,8 \%$ e $42,7 \%$.

Tabela 3 - Resumo dos dimensionamentos e percentual do peso reduzido.

\begin{tabular}{|c|c|c|c|c|c|c|c|c|c|}
\hline \multirow{2}{*}{$\begin{array}{l}\text { profun- } \\
\text { didade } \\
\text { Z } \\
(\mathrm{m})\end{array}$} & \multirow{2}{*}{$\begin{array}{l}\text { pressão } \\
\text { cálculo } \\
\mathrm{Ph} \\
(\mathrm{MPa})\end{array}$} & \multirow{2}{*}{$\begin{array}{c}\theta \\
\text { (graus) }\end{array}$} & \multicolumn{3}{|c|}{$\begin{array}{l}\text { Modelo trapezoidal proposto por } \\
\text { Ravenet }\end{array}$} & \multicolumn{3}{|c|}{$\begin{array}{l}\text { Modelo trapezoidal de Ravenet } \\
\text { Otimizado }\end{array}$} & \multirow{2}{*}{$\begin{array}{c}\text { Redução } \\
\text { do peso } \\
\text { otimizado } \\
\text { em \% }\end{array}$} \\
\hline & & & $\begin{array}{l}\mathrm{Lc} \\
(\mathrm{m})\end{array}$ & $\begin{array}{c}\mathrm{t} \\
(\mathrm{m})\end{array}$ & $\begin{array}{c}\mathrm{P} \\
(\mathrm{kN} / \mathrm{m})\end{array}$ & $\begin{array}{l}\text { Lc } \\
(\mathrm{m})\end{array}$ & $\begin{array}{c}\mathrm{t} \\
(\mathrm{m})\end{array}$ & $\begin{array}{c}\mathrm{P} \\
(\mathrm{kN} / \mathrm{m})\end{array}$ & \\
\hline 1,90 & 0,019 & 45 & 0,3000 & 0,00300 & 0,2706 & 0,2755 & 0,00210 & 0,1897 & 29,90 \\
\hline 3,90 & 0,021 & 45 & 0,3000 & 0,00400 & 0,3608 & 0,2834 & 0,00229 & 0,2064 & 42,77 \\
\hline 8,12 & 0,038 & 45 & 0,3000 & 0,00500 & 0,4509 & 0,2970 & 0,00391 & 0,3525 & 21,83 \\
\hline 25,00 & 0,042 & 45 & 0,3000 & 0,00600 & 0,5411 & 0,2970 & 0,00438 & 0,3950 & 27,01 \\
\hline
\end{tabular}


As análises anteriores foram efetuadas considerando-se a espessura como variável continua. Adicionalmente, a Figura 9 apresenta o peso otimizado resultante da imposição de valores inteiros para a espessura (em mm). Para estes casos observou-se que, de forma geral, a redução de peso ainda é bastante significativa.

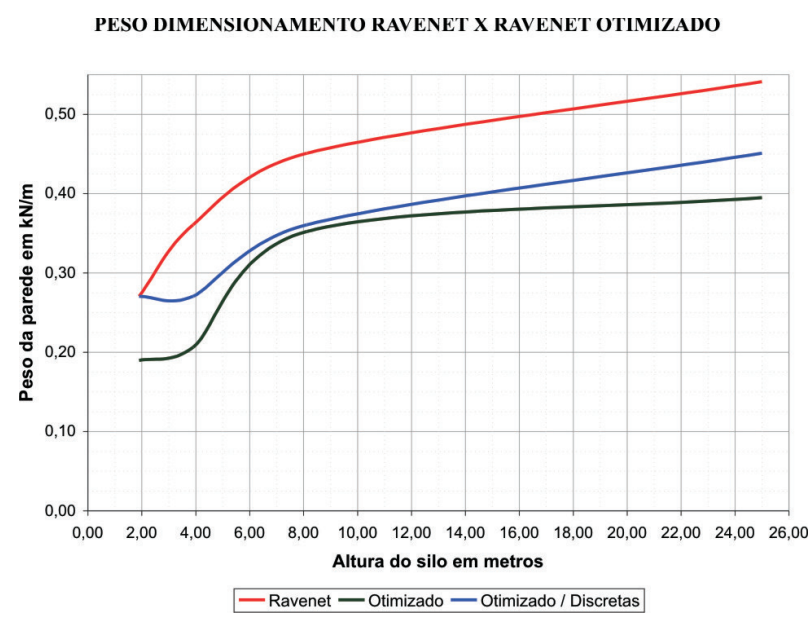

Figura 9. Peso da parede conformada com onda trapezoidal e peso otimizado.

\section{Considerações Finais}

O presente trabalho objetivou a avaliação do desempenho estrutural das paredes de silos metálicos prismáticos enrijecidas, conformadas com ondas horizontais em ziguezague e trapezoidal. Para ambas as configurações o problema foi formulado tendo como variáveis de projeto a espessura, o ângulo da inclinação do segmento da onda com a horizontal e o comprimento do segmento da onda, objetivando a minimização do peso por metro de parede.

Devido especialmente a sua ampla disponibilidade, e por se adequar ao problema proposto, optou-se pelo emprego do Microsoft Excel Solver. O mesmo mostrou-se de fácil utilização, permitindo a criação de uma ferramenta auxiliar ao projeto de silos prismáticos.
Os resultados obtidos permitiram que se confirmasse a economia obtida a partir do emprego da otimização ao problema proposto. Simulações adicionais foram efetuadas com o intuito de se verificar a variação no peso ótimo para cada uma das configurações estudadas. De forma geral, constatouse que a configuração ziguezague proporcionou estruturas mais econômicas que a configuração trapezoidal.

\section{Referências}

CALIL JUNIOR., C.; NASCIMENTO, J. W. B.; ARAÚJO, E. C. Silos metálicos multicelulares. São Carlos: EESC/USP, 1997.

GAYLORD JUNIOR, E. H.; GAYLORD, C. N. Design of steel bins for storage of bulk solids. New Jersey: Prentice-Hall Inc., 1984.

GUERRA, P. A. Silos metálicos prismáticos: otimização estrutural das paredes em seção trapezoidal e ziguezague. 2006. Dissertação (Mestrado em Engenharia) Universidade de Passo Fundo, Passo Fundo, 2006.

KRIPKA, M.; GUERRA, P. A. Otimização estrutural das paredes de silos metálicos prismáticos. In: KRIPKA, M.; CHAMBERLAIN, Z. M. (Org.). Novos estudos e pesquisas em construção metálica. Passo Fundo: UPF, 2008. p. 148-169.

RAVENET, J. Silos. Barcelona: Autor-editor, 1992.

. Teoria, investigación e construcción. Barcelona: Editores Técnicos Asociados, 1977.

TRAHAIR, N. S.'Characteristics of structural form'. In: ROTTER, J. M. (Ed.). Design of steel bins for the storage bulk solids. Sydney: University of Sydney, 1985. p. 55-57.

TROITSKY, M. S. "Tubular steel structures, theory and design”. Montreal: Concordia University, 1988.

VANDERPLAATS, G. N. Numerical optimization techniques for engineering design: with applications. New York: McGraw-Hill, 1984.

Recebido em 13 Março, 2010 - Received on March 13, 2010.

Aceito em 20 Setembro, 2010 - Accepted on September 20, 2010. 
